\title{
ESTUDO DA MAXIMIZAÇÃO DA EFICIÊNCIA DO PROCESSO EM BATELADA DE PRODUÇÃO DE ETANOL UTILIZANDO O CALDO DAS VAGENS DE ALGAROBA (Prosopis juliflora)
}

\author{
K. N. FERREIRA ${ }^{1}$, H.C. COSTA $^{1}$, M. B. MUNIZ ${ }^{2}$ e F. L. H. da SILVA ${ }^{1}$ \\ 1 Universidade Federal da Paraíba, Departamento de Engenharia Química \\ 2 Universidade Federal de Campina Grande, Departamento de Engenharia Química \\ E-mail para contato: kaline_kaline2@hotmail.com
}

RESUMO - A algarobeira é uma espécie vegetal importante no Brasil, devido a sua adaptação na região semiárida do Nordeste. No trabalho propôs-se avaliar a produção de etanol, obtido em um alambique adaptado com uma coluna de destilação com recheio, de aço inox, através da maximização da eficiência do processo de fermentação, verificando-se as influências das concentrações de leveduras e sólidos solúveis totais que foram respectivamente 11,16 e 25 (g L' $\left.{ }^{1}\right)$ e 15,20 e 25 ( ${ }^{\circ}$ Brix). Os melhores parâmetros cinéticos foram obtidos no experimento com concentração inicial de $20{ }^{\circ}$ Brix e concentração de leveduras de $16 \mathrm{~g} \mathrm{~L}^{-1}$, com produtividade de $4,83 \mathrm{~g} \mathrm{~L}^{-1} \mathrm{~h}^{-1}$, a conversão em torno de $90 \%$, o rendimento (YP/S) 0,484 g/g, no decorrer de 16 horas. O maior teor alcoólico foi $77,32 \mathrm{~g} \mathrm{~L}^{-1}$. Destilou-se $18,2 \mathrm{~L}$ do fermentado e obteve-se um volume de 1,6 L de destilado, com graduação alcoólica abaixo de $94{ }^{\circ} \mathrm{GL}$. As vagens de algaroba apresentam nutrientes para uma boa fermentação e posterior produção de bioetanol em grau químico.

\section{INTRODUÇAO}

A algarobeira é uma espécie vegetal leguminosa, não oleaginosa, nativa das regiões áridas e semiáridas, Muniz (2009), que cresce razoavelmente bem nos desertos do continente americano e em alguns desertos africanos, sendo eminentemente xerófila. As culturas nativas neste continente vão do sudoeste norte americano até a Patagônia, na Argentina. A espécie predominante no Brasil é a Prosopis juliflora, originária do deserto do Piura no Peru.

A algarobeira é uma leguminosa arbórea que concentra a maior parte do seu valor nutritivo nas vagens (frutos), constituindo-se rica fonte de carboidratos e proteínas. Durante a época do descobrimento, o uso das vagens da algaroba na alimentação humana, quando os espanhóis chegaram à América do Sul e encontraram os índios utilizando-os na alimentação, principalmente nas regiões semidesérticas, que se estendem do sul do Equador ao centro do Chile e da Argentina, Figueiredo (2004).

No aspecto econômico, Suassuna (2007) descreve que a algarobeira tem contribuído de forma decisiva para que a economia do semiárido não venha a definhar, visto que sua ausência poderia causar desequilíbrio ambiental de grandes proporções, dado ao fato de estar inserida em todas as etapas do extrativismo vegetal servindo, assim, de proteção à vegetação nativa. 
O elevado teor de açúcares, que pode chegar a $40 \%$ da massa da vagem, associado aos altos níveis de nitrogênio, favorece os processos bioquímicos e viabiliza a tecnologia de produção de bioetanol.

Neste trabalho realizou-se a caracterização físico-química e o estudo cinético da fermentação alcoólica para produção do fermentado das vagens de algaroba, posteriormente o estudo da destilação do mosto fermentado para obtenção o bioetanol hidratado.

\section{MATERIAL E MÉTODOS}

As vagens da algaroba, antes da fermentação, passaram por diversos processos para submetê-las as melhores condições de sanidade, para minimizar os riscos de contaminação do processo de fermentação, e para uma melhor extração dos açucares contidos nos vagens (fragmentação, hidratação).

A Figura 1 mostra um fluxograma dos processos realizados para produção do fermentado das vagens da algaroba

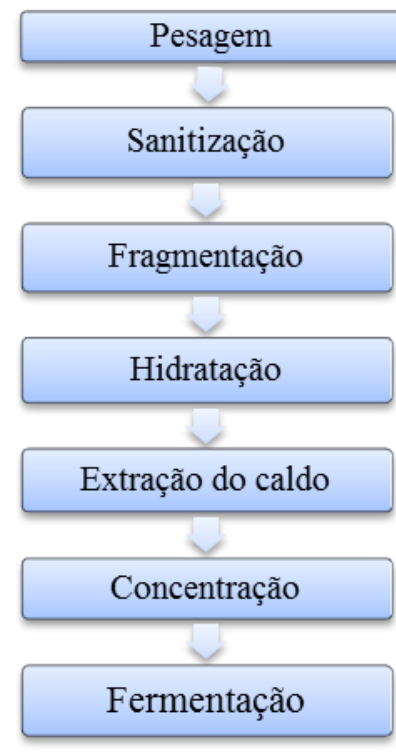

Figura 1: Fluxograma dos processos para produção do fermentado

Utilizou-se para o processo de fermentação alcoólica a levedura liofilizada Saccharomyces cerevisiae (levedura cedida pela destilaria GIASA localizada na cidade de Pedras de Fogo/PB).

Após a inoculação deu-se início ao processo de fermentação alcoólica, que foi realizado em biorreatores de polietileno utilizando-se 3 litros de caldo prensado das vagens de algaroba com suas devidas concentrações de sólidos solúveis totais (SST). Ao ser inoculada a levedura no caldo, foi retirada uma amostra de $50 \mathrm{~mL}$ de cada biorreator para realização das analises físicoquímicas (pH, Acidez Total, Temperatura, SST- ${ }^{\circ}$ Brix, Teor Alcoólico) do caldo inoculado, esse 
procedimento foi repetido a cada duas horas até o fim da fermentação. Também foram coletadas amostras de $50 \mathrm{~mL}$ de cada biorreator e essas amostras foram armazenadas em um freezer para posterior analise de biomassa e Açúcares Redutores totais (ART).

Para esse estudo cinético fez-se o uso da metodologia de planejamento fatorial $2^{2}+2$ pontos centrais utilizando-se como variáveis independentes: concentração de sólidos solúveis totais, SST ( ${ }^{\circ}$ Brix) e concentração de leveduras $\left(\mathrm{g} \mathrm{L}^{-1}\right)$ e como variáveis dependentes: \% conversão, produtividade $\left(\mathrm{g} \mathrm{L}^{-1} \mathrm{~h}^{-1}\right)$, rendimento teórico $\left(\mathrm{Y}_{\mathrm{P} / \mathrm{S}}\right)$ e rendimento em biomassa $\left(\mathrm{Y}_{\mathrm{X} / \mathrm{S}}\right)$, totalizando 6 experimentos descritos na Tabela 1.

A Tabela 1 apresenta a descrição dos experimentos para o processo de fermentação alcoólica do caldo prensado das vagens de algaroba.

Tabela 1: Matriz de planejamento fatorial para o processo de fermentação alcoólica

\begin{tabular}{|c|c|c|}
\hline Experimento & SST $\left({ }^{\circ}\right.$ Brix $)$ & Concentração de leveduras $\left(\mathbf{g L}^{-\mathbf{1}}\right)$ \\
\hline 1 & 15 & 11 \\
\hline 2 & 25 & 11 \\
\hline 3 & 15 & 21 \\
\hline 4 & 25 & 21 \\
\hline 5 & 20 & 16 \\
\hline 6 & 20 & 16 \\
\hline
\end{tabular}

Após o término da fermentação alcoólica foi realizado o processo da filtração do material fermentado (mosto) que se encontrava em repouso nos biorreatores. A separação do meio líquido do fermentado da massa celular, foi feita por centrifugação.

No final do processo foi utilizada a pasteurização com o objetivo de eliminar os microrganismos. Esse processo se deu através do fermentado dentro de garrafas pets, hermeticamente fechadas para não haver problemas de entrada de ar, água e outros materiais que interferiam nas amostras. Essas garrafas foram colocadas dentro de um pasteurizador a uma temperatura de $65 \pm 1^{\circ} \mathrm{C}$, por cerca de 30 minutos, em seguida foi dado um choque térmico, com água à temperatura ambiente $\left(25 \pm 0,5^{\circ} \mathrm{C}\right)$.

Para as aferições do $\mathrm{pH}$, o método utilizado foi o potenciométrico, com o pHmetro de bancada da marca Tecnopon modelo mPA-210, onde o mesmo foi calibrado, inicialmente, com as soluções tampões de pH 4,0; pH 7,0 e pH 9,0, Brasil (2005).

A determinação de sólidos solúveis totais foi realizada por densimetria, utilizando-se um sacarímetro de brix imergindo o mesmo em uma proveta graduada contendo $500 \mathrm{~mL}$ de caldo de algaroba e corrigindo-se a leitura obtida para a temperatura do caldo a $20{ }^{\circ} \mathrm{C}$, conforme a metodologia descrita por Brasl (2005).

A acidez total foi determinada por titulometria conforme metodologia descrita pelo Instituto Adolfo Lutz, Brasil (2005).

A concentração celular (biomassa) foi determinada adotando-se o método de massa seca que consiste em separar as células do meio, secá-las e pesá-las. A princípio, os tubos de eppendoff, com a capacidade de 1,5 mL cada, foram preenchidos com o fermentado de algaroba, 
e em seguida foram colocados no centrifugador por cerca de $10 \mathrm{~min}$, com uma rotação média de $3.000 \mathrm{rpm}$. Em seguida foi realizado o processo de lavagem do material (repetido por duas vezes). Ao término das lavagens, o material foi levado para estufa a $105{ }^{\circ} \mathrm{C}$ por $24 \mathrm{~h}$, e em seguida pesado. $\mathrm{O}$ cálculo para determinar a biomassa, foi feito por diferença entre a massa inicial (M1) e a massa final em gramas (M2), dividida pelo volume (V) da amostra utilizado (1,5 $\mathrm{mL})$, multiplicado por 1000 para obter o valor em $\mathrm{g} \mathrm{L}^{-1}$.

A metodologia empregada para determinação de açúcares redutores totais (ART) foi a do DNS (ácido 3,5 - dinitro salicílico), o aparelho utilizado para fazer as leituras foi o espectrofotômetro tipo UV, na leitura de 540nm, o método descrito por Miller (1959).

Para a obtenção do bioetanol, repetiu-se todo o procedimento da metodologia da fermentação, seguiu os dados dos pontos centrais do planejamento fatorial em uma escala maior. Fermentando 20 litros de caldo de algaroba em um reator, no final obteve-se um volume de 18,2 L de mosto fermentado, que seguiu para a coluna de destilação com recheio com a capacidade de 30 litros de volume útil.

\section{RESULTADOS E DISCUSSÃO}

$\mathrm{Na}$ Tabela 2 está expressa a composição físico-química das vagens de algaroba in natura, que se tornam importantes para um melhor conhecimento das características das vagens colhidas.

Tabela 2: Composição físico-química das vagens de algaroba in natura

\begin{tabular}{|c|c|}
\hline Análise & Composição \\
\hline Teor de Cinzas (\%) & 2,55 \\
\hline Umidade (\%) & 13,41 \\
\hline Gorduras (\%) & 2,60 \\
\hline Proteína Bruta (\%) & 8,34 \\
\hline Acidez em ácido acético $\left(\mathrm{g} \mathrm{L}^{-1}\right)$ & 0,92 \\
\hline Carboidratos (\%) & 73,07 \\
\hline Valor energético (Kcal/100g) & 349,14 \\
\hline Açúcares Totais (\%) & 39,42 \\
\hline
\end{tabular}

Observa-se, na Tabela 2, que as vagens possuem um teor significativo de açúcares totais, pouco mais de $39 \%$ de sua composição. Essa característica faz com que as vagens sejam um excelente fornecedor de substrato (açúcares) para os processos fermentativos.

Na Figura 2 está representado em um gráfico os perfis de biomassa, a cada 2 horas, relativo aos biorreatores 1 e 2 (experimentos 1 e 2), onde ambos possuem concentração inicial de levedura de $11 \mathrm{~g} \mathrm{~L} \mathrm{~L}^{-1}$, e os biorreatores 3 e 4 (experimentos 3 e 4), cujas concentração inicial de leveduras é de $21 \mathrm{~g} \mathrm{~L}^{-1}$. 


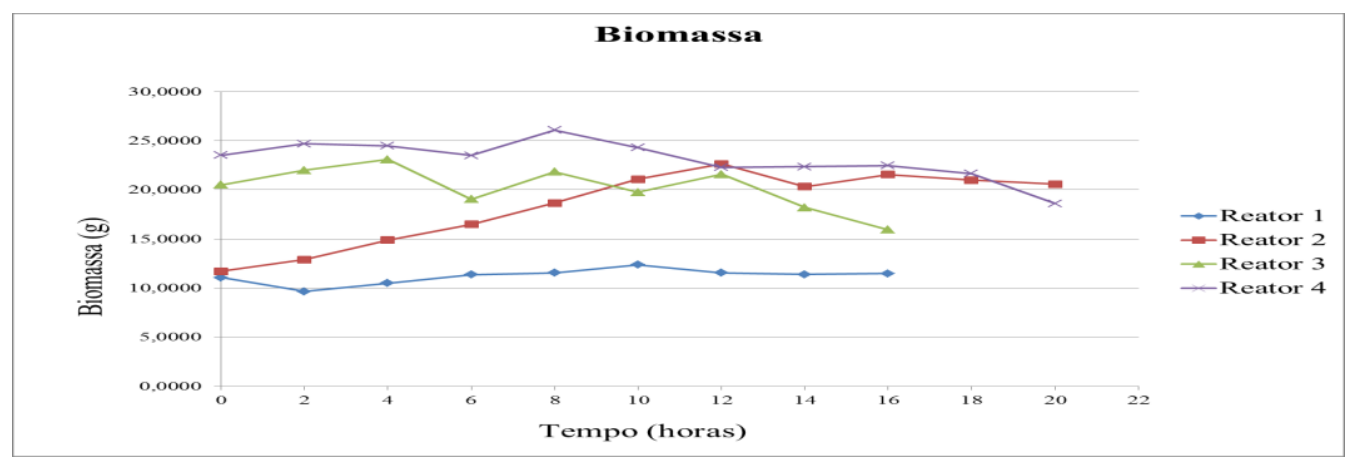

Figura 2: Comportamento do crescimento celular nos biorreatores 1, 2, 3 e 4

Observa-se pela Figura 2 que houve uma anormal variação da biomassa no decorrer do processo fermentativo, exceto para o biorreator 2, onde o crescimento celular se comportou de modo normal (aumento da massa de microrganismo com o tempo de fermentação).

Na Figura 2, observa-se que na fase exponencial de crescimento celular inicia-se no tempo de 2 horas de fermentação e atingi o seu máximo no tempo de 12 horas de fermentação.

Na Figura 3 estão representados os perfis de biomassa relativos aos pontos centrais de acordo com o planejamento fatorial mencionado que estão representados pelos biorreatores 5 e 6 , cuja concentração inicial de leveduras é de $16 \mathrm{~g} \mathrm{~L}^{-1}$ para todos.

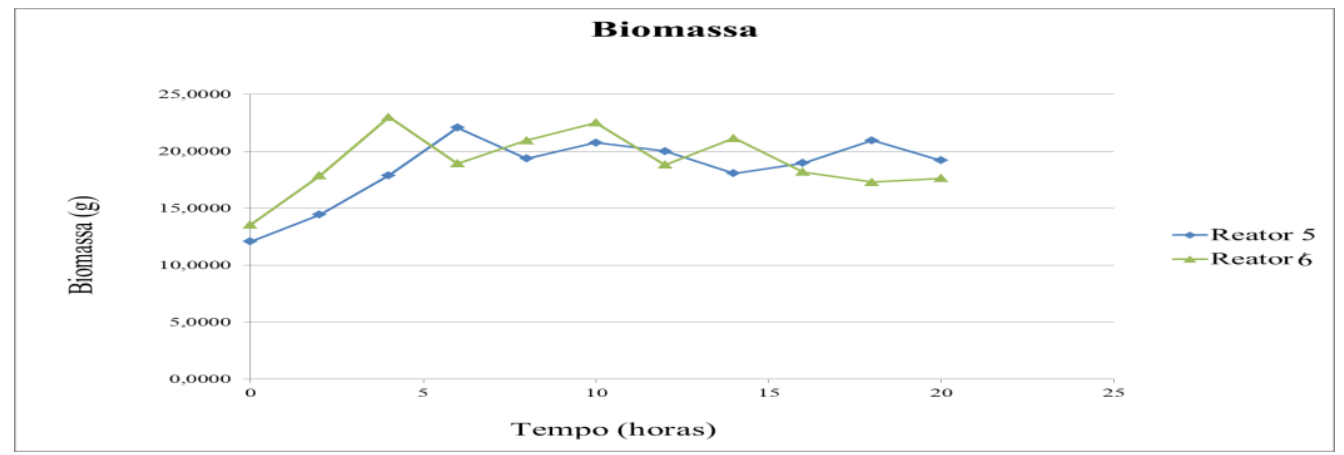

Figura 3: Comportamento do crescimento celular nos reatores 5e 6

Observa-se pela Figura 3 que o crescimento celular para os biorreatores 5 e 6 apresentou crescimento até 10 horas de fermentação, depois desse tempo houve decréscimo da massa de levedura (período de morte).

Observa-se, mais visivelmente, que a fase exponencial de crescimento celular para a fermentação em estudo iniciou-se no tempo de 6 horas de fermentação e atingiu o máximo no tempo de 10 horas e posteriormente houve o declínio até o fim da fermentação no tempo de 20 horas.

$\mathrm{Na}$ Figura 4 apresentam-se os perfis do consumo do substrato (ART) e o teor alcoólico para os biorreatores 1, 2, 3 e 4 (experimentos 1, 2, 3 e 4). 


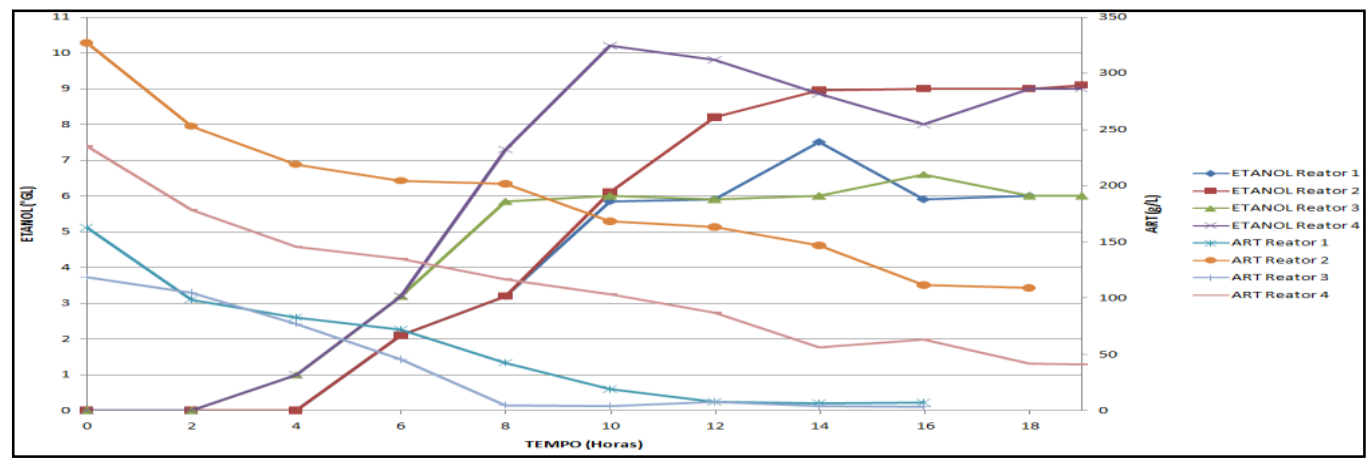

Figura 4: Consumo do Substrato versus Produção de Etanol nos biorreatores 1, 2, 3 e 4

Observa-se na Figura 4 que em todos os experimentos houve um decréscimo do substrato frente a um aumento do teor alcoólico.

Constata-se também que nos experimentos 1 e 3 as leveduras chegaram a converter praticamente todo substrato, mas esses experimentos não obtiveram os melhores valores de teor alcoólico ao final da fermentação, mas sim os menores valores, $6{ }^{\circ} \mathrm{GL}$ para ambos. Esse fato pode ser explicado pela concentração inicial de açúcares estarem nas menores concentrações, 15 ${ }^{\circ}$ Brix.

No experimento 3, observa-se também que houve um consumo muito rápido do substrato, visto que, esse experimento possuía a mais alta concentração de leveduras, $25 \mathrm{~g} \mathrm{~L}^{-1}$.

Os experimento 2 e 4 , com concentração de levedura $11 \mathrm{~g} \mathrm{~L}^{-1}$ e $21 \mathrm{~g} \mathrm{~L}^{-1}$ respectivamente, e concentração de sólidos solúveis totais $15^{\circ}$ Brix e $25^{\circ}$ Brix respectivamente, obtiveram teores alcoólicos bastante significativos ao final da fermentação, 9,1 e 9,0 ${ }^{\circ} \mathrm{GL}(\% \mathrm{v} / \mathrm{v})$ respectivamente.

Na Figura 5 estão apresentados o consumo do substrato (ART) e a produção de etanol para os biorreatores 5 e 6 (experimentos no ponto central do planejamento fatorial).

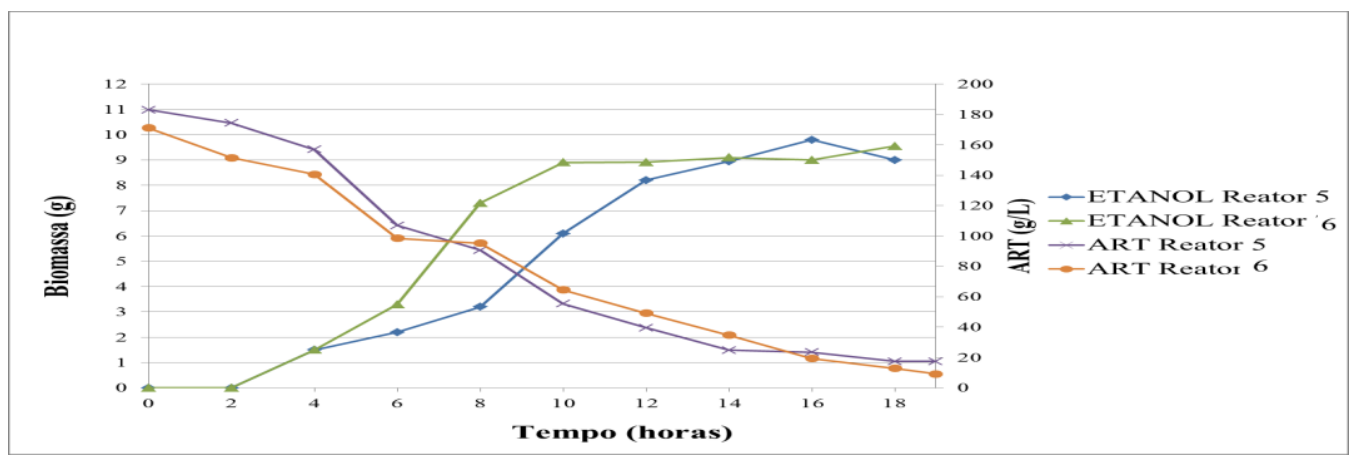

Figura 5: Consumo do Substrato versus Produção de Etanol nos biorreatores 5, 6

Observa-se pela Figura 5, que os pontos centrais - concentração de açúcares de $20 \mathrm{~g} \mathrm{~L}^{-1}$ e concentração de leveduras $16 \mathrm{~g} \mathrm{~L}^{-1}$ - apresentaram perfis semelhantes (boa reprodutibilidade do 
processo fermentativo) com o aumento da produção de etanol a medida que o substrato foi consumido.

Os parâmetros cinéticos da fermentação alcoólica (\% conversão, Produtividade, Rendimento Teórico ( $\mathrm{Y}_{\mathbf{p} / \mathbf{s}}$ ) e Rendimento em biomassa) apresentam a informação de qual experimento foi mais eficiente.

$\mathrm{Na}$ Tabela 3 estão representados os valores para os parâmetros cinéticos para cada experimento no tempo em que ocorreu o maior teor alcoólico (pico).

Tabela 3: Parâmetros cinéticos no pico de maior produção

\begin{tabular}{|c|c|c|c|c|c|c|c|c|c|}
\hline Exp. & $\begin{array}{c}\text { Tempo } \\
(\mathbf{h})\end{array}$ & $\begin{array}{c}\text { Biomassa } \\
\left(\mathbf{g ~ L}^{-1}\right)\end{array}$ & $\begin{array}{c}\text { Etanol } \\
\left({ }^{\circ} \mathbf{G L}\right)\end{array}$ & $\begin{array}{c}\text { Etanol } \\
\left(\mathbf{g ~ L}^{-1}\right)\end{array}$ & $\begin{array}{c}\mathbf{A R T} \\
\left(\mathbf{g ~ L}^{-1}\right)\end{array}$ & $\begin{array}{c}\text { Conver } \\
\mathbf{s a ̃ o} \\
(\mathbf{\%})\end{array}$ & $\begin{array}{c}\mathbf{P r o d u t}^{-1} \\
\left(\mathbf{g ~ L}^{-1} \mathbf{h}^{-1}\right)\end{array}$ & $\mathbf{Y}_{\mathbf{p} / \mathbf{s}}$ & $\mathbf{Y}_{\mathbf{x} / \mathbf{s}}$ \\
\hline $\mathbf{1}$ & 14 & 0,32 & 7,5 & 59,18 & 162,45 & 71 & 4,23 & 0,38 & 0,002 \\
\hline $\mathbf{2}$ & 20 & 1,08 & 9,1 & 71,80 & 327,07 & 43 & 3,59 & 0,33 & 0,003 \\
\hline $\mathbf{3}$ & 16 & 8,88 & 6,6 & 52,07 & 118,77 & 86 & 3,26 & 0,45 & 0,075 \\
\hline $\mathbf{4}$ & 18 & 0,78 & 9,0 & 71,01 & 234,97 & 59 & 3,94 & 0,37 & 0,003 \\
\hline $\mathbf{5}$ & 16 & 6,88 & 9,8 & 77,32 & 183,11 & 83 & 4,83 & 0,48 & 0,038 \\
\hline $\mathbf{6}$ & 18 & 3,72 & 9,5 & 74,96 & 171,12 & 86 & 4,16 & 0,47 & 0,022 \\
\hline
\end{tabular}

Pode-se observar pela Tabela 3 que os experimentos 3 e 6 foram o que apresentaram melhores \% conversão, ambos com $86 \%$ de substrato transformado em etanol. Mas o experimento 6 apresentou um maior produtividade.

Para o estudo da destilação do mosto fermentado, foi realizada uma fermentação com as condições do ponto central (20 ${ }^{\circ}$ brix e $16 \mathrm{~g} \mathrm{~L}^{-1}$ ), com 20 litros de caldo de algaroba, obtendo-se um volume de 18,2 L de mosto fermentado, que seguiu para a coluna de destilação com recheio com a capacidade de 30 litros de volume útil.

Na Tabela 4 são apresentados os dados do monitoramento da destilação.

Tabela 1: Dados do monitoramento do processo de destilação

\begin{tabular}{|c|c|c|c|c|c|c|}
\hline Destilado & Tempo de coleta & $\begin{array}{c}\text { Teor } \\
\text { alcoolico } \\
\left({ }^{\circ} \mathbf{G L}\right)\end{array}$ & $\begin{array}{c}\text { Temp. do } \\
\text { destilado } \\
\left({ }^{\circ} \mathbf{C}\right)\end{array}$ & $\begin{array}{c}\text { Temp.da } \\
\text { coluna } \\
\left({ }^{\circ} \mathbf{C}\right)\end{array}$ & $\begin{array}{c}\text { Volume de } \\
\text { destilado } \\
(\mathbf{m L})\end{array}$ & $\begin{array}{c}\text { Volume } \\
\text { de } \\
\text { álcool } \\
(\mathbf{m L})\end{array}$ \\
\hline $\mathbf{1}$ & 24 mim 15 seg & 83,0 & 30,0 & 92,0 & 297 & 246,51 \\
$\mathbf{2}$ & $50 \mathrm{mim} 56 \mathrm{seg}$ & 84,5 & 30,0 & 93,5 & 259 & 217,56 \\
$\mathbf{3}$ & 1 h 12 mim 9 seg & 70,0 & 31,0 & 94,0 & 250 & 175,00 \\
$\mathbf{4}$ & 1 h 12 mim 13 seg & 34,0 & 40,0 & 94,0 & 190 & 64,60 \\
$\mathbf{5}$ & 1 h 12 mim 17 seg & 34,0 & 40,0 & 94,0 & 68 & 23,12 \\
$\mathbf{6}$ & 1 h 12 mim 20 seg & 34,0 & 32,0 & 100,0 & 40 & 13,60 \\
\hline
\end{tabular}


Observa-se na Tabela 4 que com o aumento do tempo de destilação ocorreu uma diminuição na graduação alcoólica do destilado (produto). A coluna de destilação de alambique acoplada a uma coluna de recheio de bolas de vidro, ainda precisa ser melhor operada, pois o teor alcoólico do etanol hidratado (objetivo do trabalho), deve ser, de no mínimo, acima de $94{ }^{0} \mathrm{GL}$.

\section{CONCLUSÕES}

A concentração máxima de etanol produzida no fermentado das vagens de algaroba foi de 9,8 ${ }^{\circ} \mathrm{GL}$ obtido quando se operou ( $20^{\circ}$ brix e $16 \mathrm{~g} \mathrm{~L}^{-1}$ ). A destilação do fermentado do caldo (vinho) da algaroba para a produção de bioetanol não foi eficiente, precisando estudar a otimização do processo de destilação, utilizando-se do alambique de cobre acoplado a coluna de recheio de bolas de vidro para conseguir etano hidratado dentro do teor alcoólico preconizado pela legislação vigente (em torno de $94{ }^{\circ} \mathrm{GL}$ ).

\section{REFERÊNCIAS}

BRASIL. Ministério da Saúde. Agência Nacional de Vigilância Sanitária. Métodos Fisicoquímicos para análises de alimentos. Brasília: Ministério da Saúde, 2005. 1018p. (Série A. Normas Técnicas).

FIGUEIREDO, A.A.; ASCHERI, J.L.R.; CARVALHO, C.W. Produção de expandidos à base de farinha mista de algaroba e arroz e de algaroba e milho. In: Congresso Brasileiro de Ciência e Tecnologia de Alimentos - Estratégia para o Desenvolvimento, 2004, Recife. Anais...Recife: SBCTA, 2004. CD-Rom.

MILLER, G. Use of dinitrosalicilic acid reagent for determination of reducing sugars. Analytical Chemistry, v.31, p. 426-428. 1959.

MUNIZ, M.B.; Processamento das vagens de algaroba para produção de bioprodutos; Dissertação (Doutorado) Universidade Federal de Campina Grande, Campina Grande, PB, 2009.

SUASSUNA, J. Algarobeira - produção e produtividade. Recife, 2007, 6p. Boletim da Empresa Brasileira de Pesquisa Agropecuária EMBRAPA, 2007. 